\title{
Clinico-pathological considerations in a 48- years-old female with acute kidney injury: is it lupus nephritis, ANCA-associated vasculitis or something else?
}

\author{
Marie Lemerle ${ }^{1,2}$, Anne-Sophie Garnier ${ }^{1,2}$, Anne Croue ${ }^{3}$, Alain Chevailler ${ }^{4}$, Jean-Paul Saint-André ${ }^{2,4}$,
} Jean-François Subra ${ }^{1,2}$, Jean-François Augusto ${ }^{1,2}$ and Julien Demiselle ${ }^{1,2^{*}}$ (D)

\begin{abstract}
Background: The value of ANCA positivity in the setting of systemic lupus erythematous and their pathogenicity remains uncertain.

Case presentation: We report the case of a 48-year-old female with rapidly progressive kidney failure, arthromyalgia and weight loss. Auto-immune screening showed anti-dsDNA antibodies, complement consumption and triple ANCA positivity. A first kidney biopsy done at presentation highlighted class IV-G glomerulonephritis with elective extra-capillary involvement and mainly C1q glomerular deposition at immunofluorescence study. After three months of a regimen combining steroids and cyclophosphamide, a second biopsy was performed and showed class IV-G glomerulonephritis with mainly endocapillary proliferation.

Conclusion: This case is atypical in view of immunological profile and kidney histopathological presentation and evolution and gives rise to discussion in view of recent data on ANCA value in lupus nephritis, and suggests that different auto-immune pathways may be involved in lupus nephritis.
\end{abstract}

Keywords: Lupus glomerulonephritis, ANCA, Immunology

\section{Background}

Several lines of evidence indicate that anti-neutrophil cytoplasmic antibodies (ANCAs), especially myeloperoxidase (MPO)-ANCAs, are pathogenic auto-antibodies in ANCAs-associated vasculitis (AAV) [1]. However, ANCAs can also be detected in healthy subjects and in a number of inflammatory/auto-immune diseases where their pathophysiological significance remains debatable [2].

Detection of ANCAs at diagnosis or in the course of systemic lupus erythematosus (SLE) is quite common, reported in up to $20 \%$ of patients [3]. According to literature, perinuclear ANCAs (p-ANCAs) rather than cytoplasmic ANCAs (c-ANCAs) are detected using indirect immunofluorescence (IIF) in SLE [3, 4].

\footnotetext{
* Correspondence: julien.demiselle@chu-angers.fr

'Service de Néphrologie-Dialyse-Transplantation, Université d'Angers, CHU

Angers, 4 rue Larrey, 49033 Angers CEDEX 09, Angers, France

${ }^{2}$ LUNAM Université, Angers, France

Full list of author information is available at the end of the article
}

However, the significance of these ANCAs is quite variable among SLE patients, some of them having ANCAs directed towards minor ANCA antigens (ie, lactoferrin, BPI, elastase.), and others towards major ANCA antigens (MPO or PR3), the latters' being those associated with AAV [3]. Even if ANCA positivity has been assoiated with higher disease activity in SLE patients, their potential role in organ injury still have to be demonstrated [3].

Recently, Tuner-Stokes et al. studied retrospectively more than 200 kidney biopsies from patients with lupus nephritis (LN) and compared histopathological features of those associated with ANCAs at biopsy to those without [4]. As already reported in past studies, a predominance of p-ANCA of MPO specificity was observed in SLE patients [3]. More interestingly, they found that patients with ANCA positivity had more frequently diffuse glomerulonephritis with segmental involvement (class 
IV-S LN ISN/RPS classification) and lesions of glomerular necrosis, as compared to patients without ANCAs. Moreover, ANCA positive patients had a worse kidney function at the time of biopsy, higher anti-dsDNA antibody levels and lower complement levels. Thus, ANCAs positivity was associated with a specific kidney histological and biological phenotype in SLE patients. These observations allow to reopen an old debate on the potential pathogenic action of ANCAs in LN [5].

Here, we report the case of a 48-years old female referred to our department with systemic symptoms and acute kidney injury. The clinical and biological presentation was very suggestive of SLE, but strong ANCA positivity was also detected. The kidney histology at admission and its evolution 4 months after treatment initiation was uncommon and gives rise to discussion.

\section{Case presentation}

A 48-years old Asian female was referred to our hospital with a recent history of weakness, myalgia and arthralgia. She also complained of anorexia with $3 \mathrm{~kg}$ weight loss. She had no past medical history and was free of any medication on admission. At presentation, blood pressure was $180 / 80 \mathrm{mmHg}$, heart rate was $91 / \mathrm{min}$, temperature was normal. Heart and lung auscultation, as well as abdominal examination, were normal. She had no skin involvement nor lymphadenopathy or synovitis.

Biology showed acute kidney injury with serum creatinine at $209 \mu \mathrm{mol} / \mathrm{L}$. White blood cell count $(4.8 \mathrm{G} / \mathrm{L})$ and platelet count $(269 \mathrm{G} / \mathrm{L})$ were normal, but anemia with hemoglobin at $7.8 \mathrm{~g} / \mathrm{dL}$ was present. C-reactive protein was slightly increased $(14 \mathrm{mg} / \mathrm{dL})$. Urinalysis revealed glomerular proteinuria (proteinuria to creatinine ratio $(\mathrm{P} / \mathrm{C}) 3.6 \mathrm{~g} / \mathrm{g}$ ) and microscopic hematuria. Kidney ultrasound examination showed normal sized kidneys and excluded obstruction. Thus, we concluded to acute glomerular syndrome and performed immunological laboratory tests. Antinuclear antibodies (ANA) (1/2560), as well as anti-dsDNA (292 UI/mL), anti-SSA and antiSSB antibodies were detected. Type 3 cryoglobulinemia and complement consumption (decreased $\mathrm{C} 3, \mathrm{C} 4$ and $\mathrm{CH} 50$ ) were also present. Search for lupus anticoagulant, IgG anti-cardiolipin and anti-beta-2GP1 antibodies was negative. Finally, pANCA were detected at 1/2000 titer using IIF. ELISA showed the concomitant presence of MPO, PR3 and lactoferrine ANCAs with a strong positivity for MPO and lactoferrine ANCAs and a low positivity for PR3 ANCAs. ANCA detection using multiplex technology also detected MPO ANCA at high level, and low PR3 ANCA level.

LN was first considered leading us to perform a kidney biopsy. On optical examination, extra-capillary circumferential cellular or fibro-cellular crescents were observed in 10 of 16 glomeruli. Segmental endocapillary proliferation was absent to very modest, without any lesions of capillary necrosis (Fig. 1). Immunofluorescence analysis showed strong and diffuse mesangial and parietal $\mathrm{C} 1 \mathrm{q}$ staining with a granular deposition pattern, while IgG, IgA, IgM, and C3 deposits were quite limited, of mesangial topography and only segmental (Fig. 1).

Following biopsy, an immunosuppressive treatment was initiated with an association of steroids, hydoxychloroquine and pulse intravenous cyclophosphamide (CYC, $500 \mathrm{mg}$ every two weeks) according to the EuroLupus protocol trial [6]. Under this regimen, a progressive improvement of both her general condition and kidney function was observed. At month 3 from treatment initiation (after the 6th CYC injection), serum creatinine was $116 \mu \mathrm{mol} / \mathrm{L}$ and $\mathrm{P} / \mathrm{C}$ ratio decreased to $0.50 \mathrm{~g} / \mathrm{g}$. Antinuclear antibodies decreased to $1 / 200$, anti-dsDNA antibodies became undetectable, and complement returned within normal range. ANCAs were still detectable although at lower titer using IIF $(1 / 200)$, with only MPO ANCAs remaining slightly positives at ELISA and multiplex assays.

At that time, we decided to perform a systematic kidney biopsy to analyze histological response to treatment. On optical examination, only fibrotic crescents were observed in 9/14 glomeruli, none of them being cellular of fibro-cellular. Global lesions of endocapillary proliferation were observed in most glomeruli, without lesions of capillary necrosis (Fig. 2). Immunofluorescence analysis showed diffuse mesangial and parietal $\mathrm{C} 1 \mathrm{q}$ staining at a lower intensity as compared to the diagnostic biopsy. IgG, IgA, IgM, and C3 deposits remained limited, in their pattern and intensity (Fig. 2).

After the biopsy results, we decided to continue CYC. However, after the 8th CYC injection, the patient developed toxidermia which we attributed to CYC. At that time, we decided to start mycophenolate mofetil (MMF) at $1.5 \mathrm{~g} /$ day.

One year after initial admission and under MMF for 12 months, she has no clinical manifestation of SLE and did not relapse nephritis. Renal function returned to near normal values (serum creatinine of $90 \mu \mathrm{mol} / \mathrm{L}$, MDRD eGFR $60 \mathrm{~mL} / \mathrm{min} / 1.73 \mathrm{~m}^{2}$ ). ANCA detection was negative on IIF evaluation and ELISA, with anti-MPO being still detectable at very low level using multiplex assay. ANA were stable at $1 / 200$, complement was in normal range and search for cryoglobulin was negative.

\section{Discussion and conclusion}

The present case represents a very uncommon overlap presentation of SLE and AAV according to both its immunological and kidney pathological aspects. First, our patient presented with a typical SLE serology, but had also circulating auto-antibodies towards three different ANCA antigens, which is an extremely rare condition. Second, the kidney pathological lesions observed at 

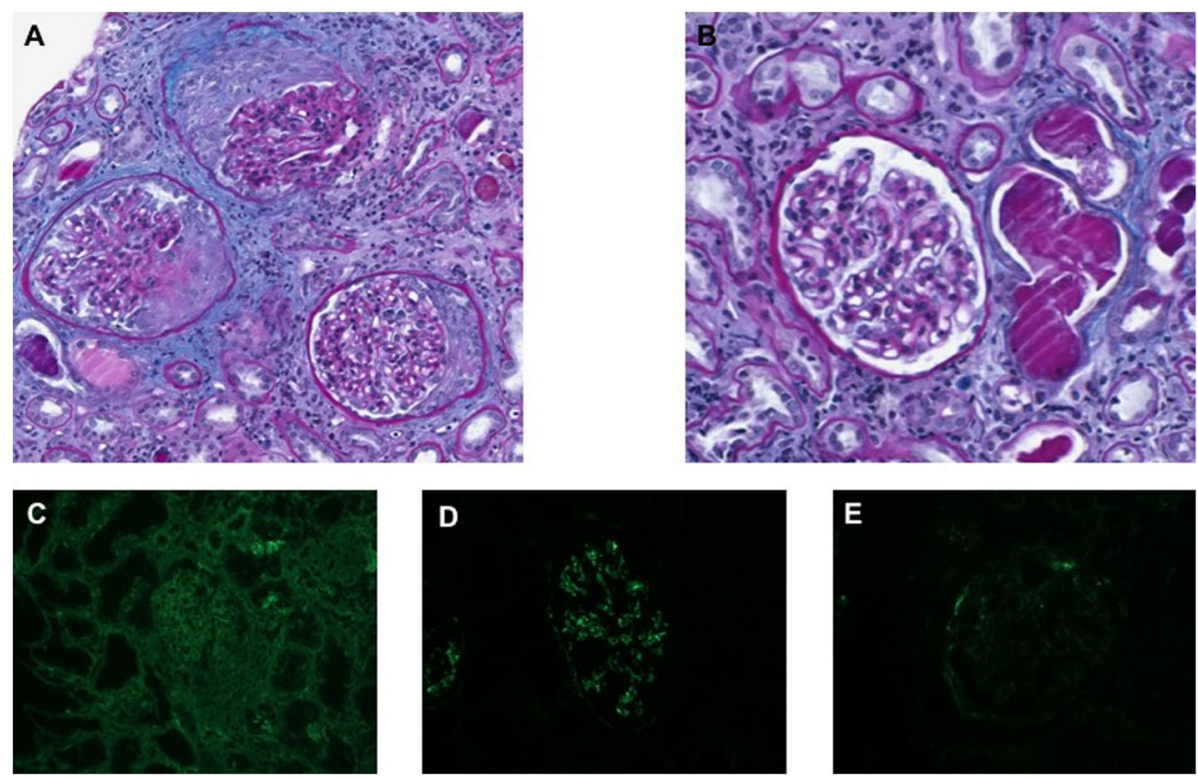

Fig. 1 Kidney biopsy done at admission. (a,b) Light microscopy examination showing (a) cellular circumferential crescents within 3 glomeruli, and (b) a normal glomeruli. Periodic acid-Schiff staining. (c, d, e), Immunofluorescence analysis showing predominant C1q staining (d), as compared to $\lg G(\mathbf{c})$ and $C 3(\mathbf{e})$ staining

diagnosis, as well as their evolution on control biopsy, were uncommon in comparison to those usually observed in proliferative forms of LN [7, 8]. The present case illustrates the complexity of immune mechanisms acting in SLE, and raises the question of the importance of histology findings in the choice of treatment in such situations.
The first atypical aspect in this observation is the coexistence of a typical SLE serology (ANA, anti-dsDNA ab, complement consumption) and ANCA positivity. As mentionned above, ANCAs, mainly p-ANCAs of MPO specificity, are detected in up to $20 \%$ of SLE patients, which allows to conclude that it is finally a quite common condition. However, in our patient, ANCAs directed towards
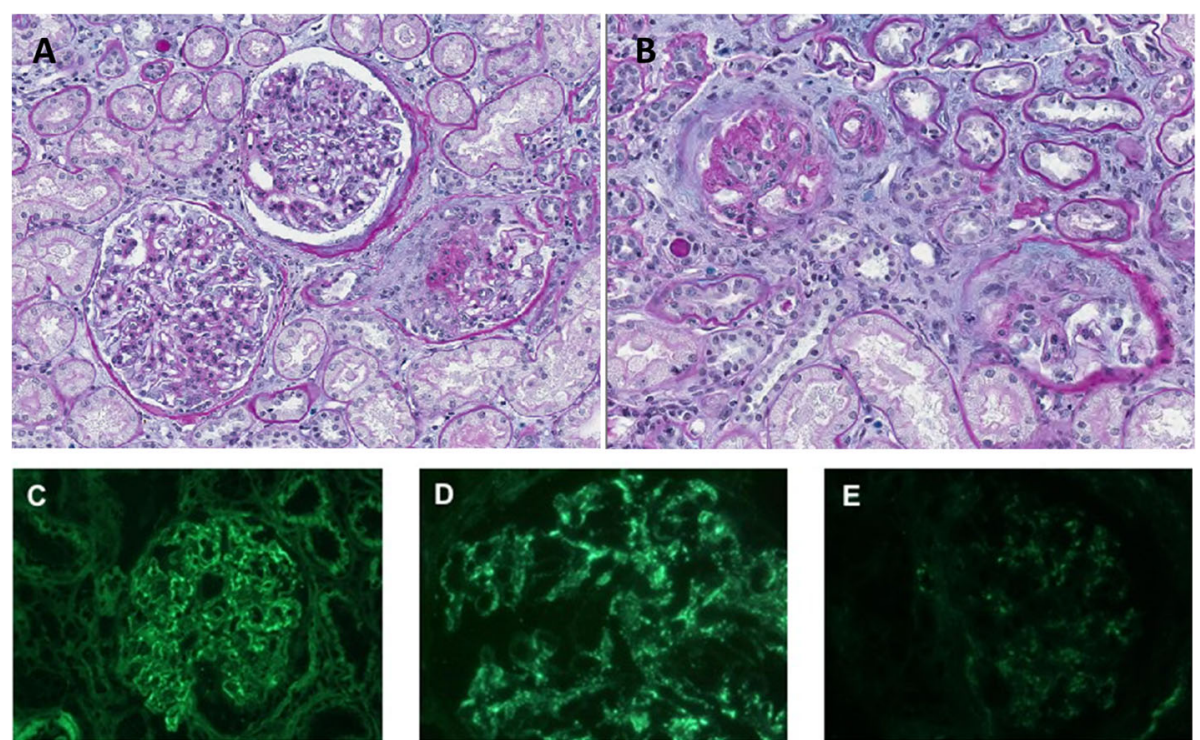

Fig. 2 Control kidney biopsy done at month 4 from admission. (a,b) Light microscopy examination showing (a) diffuse endocapillary proliferation in 2 glomeruli, and (b) fibrotic crescents in 2 glomeruli. Periodic acid-Schiff staining staining. (c, d, e), Immunofluorescence analysis showing predominant C1q staining (d), as compared to $\operatorname{lgG}(\mathbf{c})$ and C3 (e) staining 
three different ANCA antigens, namely MPO, PR3 and lactoferrin, were detected. According to the persistence of p-ANCA pattern while anti-dsDNA disappeared, a cross reactivity anti-dsDNA was unlikely [9]. This condition represents a quite exceptional situation that may be related to particular auto-immune pathways acting in our patient. In contrast with double positive ANCAs, directed towards a major ANCA antigen (MPO or PR3) and towards a minor ANCA antigen, that is finally frequently observed (notably in AAV), ANCA positivity towards both MPO and PR3 appears to be extremely rare [10, 11]. Indeed, in a past study, only 23 patients with this phenotype among 3095 consecutive ANCA positive patients, representing less than $1 \%$ of patients [12]. Interestingly, most patients with this condition had inflammatory or auto-immune diseases including SLE, but none had AAV. Confirming the rarity of this association, in a recent study analyzing ANCA positivity value in LN patients, double $\mathrm{MPO} / \mathrm{PR} 3$ positivity was observed in $3 / 32$ patients with ANCA positivity among 254 consecutives $\mathrm{LN}$ patients, thus randomly $1 \%$ of LN patients [4].

The second atypical aspect of our observation is kidney histopathology at both first and second biopsy. According to the International Society of Nephrology (ISN) / Renal Pathology Society (RPS), the first renal biopsy of our patient responded to the definition of class IV (diffuse, lesions involving $>50 \%$ of glomeruli), subtype class IV-G (global, lesions affecting $>50 \%$ of the glomerular tuft) [7]. Among class IV-G LN, crescentic forms involving more than $50 \%$ of glomeruli, where crescents take up over half space in Bowman's capsule, have been poorly analyzed. Yu et al. observed that these crescentic forms represented $20 \%$ of 152 class IV-G LN consecutive patients [13]. Interestingly, as compared to noncrescentic one's, all patients with crescentic class IV-G LN forms presented with rapidly progressive glomerulonephritis (RPGN) and had a poorer renal survival, with higher rates of relapse at 4 years from initial diagnosis. Moreover, while endocapillary proliferation was comparable between groups, crescentic forms were associated with significantly lesser immune complex deposition at immunofluorescence study. ANCA positivity (mainly MPO ANCAs) at biopsy was present in 30\% of patients with crescentic form, which was significantly higher as compared to the $2.5 \%$ observed in patients with noncrescentic class IV-G LN. These observations show that SLE patients with ANCAs are more likely to present with crescentic LN and thus suggest a role for ANCAs in crescent development. As compared to the study of $\mathrm{Yu}$ et al., two particular aspects deserve to be emphasized in our case. First, endocapillary proliferation was only very limited in our patient. Second, immunofluorescence study showed an unbalanced deposition of immune complexes. Indeed, strong and diffuse $\mathrm{C} 1 \mathrm{q}$ deposition was present, in contrast to other immune complexes that were mildly represented with only segmental deposition. It is tempting to link this atypical LN presentation to ANCA positivity by suggesting a direct pathogenic role of ANCAs or the involvement of specific auto-immune pathways related to the uncommon tolerance breakdown toward 3 different ANCA antigens observed in our patient.

This overlap between AAV and LN suggests common pathophysiological aspects. One of them could be related to Neutrophil Extracelular Traps (NETs) formation, with a different manner between AAV and LN in NETs formation (lytic versus non lytic formation) [14].

The analysis of the second biopsy is also of great interest. Indeed, all crescents became fibrotic, showing the efficiency of immunosuppressive treatment on the extracapillary lesions. On immunofluorescence evaluation, immune complex deposits were comparable to what was observed on the first biopsy. However, and surprisingly, the endocapillary proliferation became diffuse and global, thus much more pronounced as compared to the first biopsy. It allowed to classify it into IV-G subtype, but no longer because of crescentic involvement. Finally, the present case report showed conversion from crescentic class IV-G to endocapillary proliferative class IVG. Thus, it shows that lesions can vary between crescentic and endocapillary proliferation forms in the same patient and we suggest that this may be related to modulation of involved pathogenic pathways under immunosuppressive treatment. Interestingly, such observation of transitions between LN classes have been previously reported, especially between class IV-G and IV-S [15]. Biological determinants of these changes remain to be elucidated.

The understanding of biological pathways modulating the histological phenotype of LN may translate into more targeted and efficient therapies. In our patient, given the predominant crescentic presentation at first biopsy, we decided to initiate CYC rather than MMF as induction regimen. We speculated that, even if no fibrinoid necrosis was observed, maybe pathways common to those involved in AAV were acting. We observed a good response of the crescentic lesions to steroid/CYC regimen. Next, at the second biopsy showing mainly endocapillary proliferation, given CYC-related toxidermia, we opted rather to MMF while we were considering to start rituximab before biopsy as used in maintenance treatment of AAV. However, we must acknowledge that all these considerations remain speculatives because some reports have also suggested a better improvement of crescentic LN forms under MMF as compared to CYC [16].

This atypical case report highlights the complexity of LN pathophysiology. Transition from crescentic to endocapillary proliferative forms, which has to our knowledge 
not been reported previously, suggests that different autoimmune pathways may be involved at different extend and at different timings in the same patient. It also highlights the importance of more systematic kidney biopsy to better understand the pathophysiology of LN, especially in patients with atypical histological and immunological presentations as it was the case in our patient.

\section{Abbreviations}

AAV: ANCA associated vasculitis; ANA: Antinuclear antibodies; ANCA: Antineutrophil cytoplasmic antibodies; AntidsDNA: Anti double strain DNA antibody; CYC: Cyclophosphamide; ISN: International Society of Nephrology; LN: Lupus nephritis; MMF: Mycophenolate Mofetil; MPO: Myeloperoxydase; NETs: Neutrophil Extracellular Traps; PR3: Proteinase 3; RPGN: Rapidly progressive glomerulonephritis; RPS: Renal Pathology Society; SLE: systemic lupus erythematosus

\section{Acknowledgements}

Not applicable.

\section{Authors' contributions}

$M L, J D$, JFA drafted the manuscript, ASG and JFS revised the manuscript, AC and JPSA performed histological analysis of biopsies and revised the manuscript, AChev performed immunologic analysis and revised the manuscript. All the authors have read and approve the final version of the manuscript.

\section{Funding}

Not applicable.

\section{Availability of data and materials}

Not applicable.

\section{Ethics approval and consent to participate} Not applicable.

\section{Consent for publication}

Written informed consent was obtained from the patient for publication of this Case Report and any accompanying images. A copy of the written consent is available for review by the Editor of the journal.

\section{Competing interests}

All the authors declare to have no competing interest.

\section{Author details}

'Service de Néphrologie-Dialyse-Transplantation, Université d'Angers, $\mathrm{CHU}$ Angers, 4 rue Larrey, 49033 Angers CEDEX 09, Angers, France. ${ }^{2}$ LUNAM Université, Angers, France. ${ }^{3}$ Département de Pathologie Cellulaire et Tissulaire, Université Angers, CHU Angers, Angers, France. ${ }^{4}$ Laboratoire d'Immunologie, Université d'Angers, CHU Angers, Angers, France.

Received: 12 January 2019 Accepted: 20 August 2019

Published online: 27 August 2019

\section{References}

1. Jennette JC, Falk RJ, Hu P, et al. Pathogenesis of antineutrophil cytoplasmic autoantibody-associated small-vessel vasculitis. Annu Rev Pathol. 2013;8: 139-60. https://doi.org/10.1146/annurev-pathol-011811-132453.

2. Beauvillain C, Delneste $Y$, Renier $G$, et al. Antineutrophil cytoplasmic autoantibodies: how should the biologist manage them? Clin Rev Allergy Immunol. 2008:35:47-58. Research Support, Non-U.S. Gov't Review 2008/01/08. https://doi.org/10.1007/s12016-007-8071-9.

3. Sen D and Isenberg DA. Antineutrophil cytoplasmic autoantibodies in systemic lupus erythematosus. Lupus 2003; 12: 651-658. 2003/09/30. DOl: https://doi.org/10.1191/0961203303/u456rr.

4. Turner-Stokes T, Wilson HR, Morreale M, et al. Positive antineutrophi cytoplasmic antibody serology in patients with lupus nephritis is associated with distinct histopathologic features on renal biopsy. Kidney Int 2017; 92: 1223-1231. 2017/07/29. DOl: https://doi.org/10.1016/j.kint.2017.04.029.
5. Weinstein A. ANCA in lupus nephritis. Lupus. 2018;27:522. 2017/10/24 https://doi.org/10.1177/0961203317734925.

6. Houssiau FA, Vasconcelos C, D'Cruz D, et al. Immunosuppressive therapy in lupus nephritis: the Euro-Lupus Nephritis Trial, a randomized trial of lowdose versus high-dose intravenous cyclophosphamide. Arthritis Rheum 2002; 46: 2121-2131. 2002/09/05. DOI: https://doi.org/10.1002/art.10461.

7. Weening JJ, D'Agati VD, Schwartz MM, et al. The classification of glomerulonephritis in systemic lupus erythematosus revisited. J Am Soc Nephrol 2004; 15: 241-250. 2004/01/30.

8. Wilhelmus S, Alpers CE, Cook HT, et al. The Revisited Classification of GN in SLE at 10 Years: Time to Re-Evaluate Histopathologic Lesions. J Am Soc Nephrol 2015; 26: 2938-2946. 2015/07/15. DOl: https://doi.org/10.1681/ASN.2 015040384.

9. Jethwa HS, Nachman PH, Falk RJ, et al. False-positive myeloperoxidase binding activity due to DNA/anti-DNA antibody complexes: a source for analytical error in serologic evaluation of anti-neutrophil cytoplasmic autoantibodies. Clin Exp Immunol 2000; 121: 544-550. 2000/09/06.

10. Talor MV, Stone JH, Stebbing J, et al. Antibodies to selected minor target antigens in patients with anti-neutrophil cytoplasmic antibodies (ANCA). Clin Exp Immunol. 2007;150:42-8. https://doi.org/10.1111/j.1365-2249.2007. 03453.x.

11. Simon A, Subra JF, Guilpain P, et al. Detection of anti-Pentraxin-3 autoantibodies in ANCA-associated Vasculitis. PLoS One. 2016;11:e0147091. https://doi.org/10.1371/journal.pone.0147091.

12. Denomme AS, Lakomy D, Olsson N, et al. Patients with dual MPO- and PR3ANCA do not present primary systemic necrotizing vasculitis. Joint Bone Spine. 2015;82:292-294. 2015/01/28. https://doi.org/10.1016/j.jbspin.2014.11.004.

13. Yu F, Tan Y, Liu G, et al. Clinicopathological characteristics and outcomes of patients with crescentic lupus nephritis. Kidney Int 2009; 76: 307-317. 2009/ 05/01. DOI: https://doi.org/10.1038/ki.2009.136.

14. Van Dam LS, Rablink TJ, Van Kooten C, et al. Clinical implications of excessive neutrophil extracellular trap formation in renal autoimmune diseases. Kidney Int Reports 2019; 4: 196-211.2018/11/12. DOl: https://doi. org/10.1016/j.ekir.2018.11.005

15. Hill GS, Delahousse M, Nochy D, et al. Class IV-S versus class IV-G lupus nephritis: clinical and morphologic differences suggesting different pathogenesis. Kidney Int. 2005;68:2288-2297. 2005/10/14. https://doi.org/1 0.1111/j.1523-1755.2005.00688.x.

16. Tang Z, Yang G, Yu C, et al. Effects of mycophenolate mofetil for patients with crescentic lupus nephritis. Nephrology (Carlton) 2008; 13: 702-707. 2008/09/06. DOl: https://doi.org/10.1111/j.1440-1797.2008.00975.x.

\section{Publisher's Note}

Springer Nature remains neutral with regard to jurisdictional claims in published maps and institutional affiliations.
Ready to submit your research? Choose BMC and benefit from:
- fast, convenient online submission
- thorough peer review by experienced researchers in your field
- rapid publication on acceptance
- support for research data, including large and complex data types
- gold Open Access which fosters wider collaboration and increased citations
- maximum visibility for your research: over $100 \mathrm{M}$ website views per year
At BMC, research is always in progress.
Learn more biomedcentral.com/submission 